\title{
Editorial
}

\section{Circulating markers in metastatic cancer: new perspectives}

\author{
Irene A. George ${ }^{1,2, \dagger}$, Janani Sambath ${ }^{1,2, \dagger}$, Prashant $\operatorname{Kumar}^{1,2,3, *}$ \\ ${ }^{1}$ Institute of Bioinformatics, International Technology Park, 560066 Bangalore, Karnataka, India, ${ }^{2}$ Manipal Academy of \\ Higher Education (MAHE), 576104 Manipal, Karnataka, India, ${ }^{3}$ Somaiya Institute of Research and Consultancy \\ (SIRAC), Somaiya Vidyavihar University (SVU), 400077 Mumbai, Maharashtra, India
}

\section{TABLE OF CONTENTS}

\author{
1. Author contributions \\ 2. Ethics approval and consent to participate \\ 3. Acknowledgment \\ 4. Funding \\ 5. Conflict of interest \\ 6. References
}

Metastasis is one of the leading causes of cancerrelated death resulting from the final outcome of multiple processes such as epithelial-mesenchymal transition (EMT) and the reverse (MET), extracellular matrix (ECM) remodeling, immune system activation and reprogramming of the tumor microenvironment. This special issue on "Noninvasive strategies in metastatic dissemination" focuses on recent advances and novel studies in exosomes as well as other cellular processes influencing metastasis in cancer. The special issue highlights the areas covering novel insights into the role of phenotypic plasticity and EMTassociated heterogeneity in circulating tumor cells (CTCs), exosomes in cancer development and ECM remodeling in tumor progression and metastasis.

Intercellular communication via the cargoes carried by exosomes is a key facilitator to bridge between the primary tumor and the tumor microenvironment in metastasis. Extracellular vesicles (EVs) released by cells contain biomolecules involved in pro-tumorigenic processes. Recent studies support the role of EVs in promoting cancer incidence, progression and organ-specific metastasis. The comprehensive review by Visan et al. [1] covers the role of exosomes in EMT. In this article, the authors survey many aspects of EMT, metastasis, the mechanism of exosomemediated EMT and the role of exosomes in conferring therapy resistance [1]. The article by Carvalho et al. [2] further discusses the potential of utilizing the EV proteome for biomarker profiling. The authors compared the EV proteome and cellular proteome from the NCI-60 human tumor cell line panel to identify the expression of cancer hallmark proteins. This meta-analysis suggests high abundance of cancer hallmark proteins in EV proteomes and a potential role for predicting tumor tissue of origin, cancer diagnostics and prognostics [2].

The next intrinsic cellular phenomenon that drives metastasis and cancer invasion is the epithelial to mesenchymal transition (EMT) which has been classified into three different biological subtypes based on the biological process: Type 1 EMT is involved in developmental processes, type 2 EMT in wound healing, tissue regeneration and inflammation, and type 3 is associated with metastasis and progression of cancer. The primary difference between the developmental EMT and cancer-associated EMT is the lack of molecular regulation in the latter. Dhar et al. [3] describe the physiologically controlled pseudo-malignant behavior of the trophoblast during development. The authors also describe the role of hypoxia, ECM and signaling cascades in the trophoblast EMT and invasion, and further compare it with type 3 EMT [3]. Understanding the molecular regulation of trophoblast EMT could further understanding the molecular mechanisms of cancer-related EMT. In another report on EMT, Roy et al. [4] further explain the transcriptional regulation of EMT and investigate the role of type 3 EMT in stemness and plasticity. Raja et al. [5] review recent evidence on the role of EMT in cell survival, immune evasion, resistance to anoikis and metastasis. Circulating tumor cells (CTCs) entering into the bloodstream through intravasation involving EMT results in extravasation and metastatic colonization. Thus, the deeper characterization of CTCs will provide an opportunity to understand the mechanism of metastasis in solid tumors. The review focused on the dynamic state of EMT and plasticity signatures across CTCs datasets. The review also discussed the importance of an intermediate phenotype and its 
correlation with cellular plasticity [5]. Recent studies provide evidence of the role of oncogenic miRNAs as EMT promoters by repressing epithelial characteristics and tumor suppressive signaling pathways. Kumar et al. [6] summaries studies on the crosstalk between miRNA, oncogenic signaling pathways and EMT in breast cancer progression, concluding that miRNAs play a vital role in tumor initiation, progression and metastasis. The review further considers the importance of circulating miRNA profiling from CTCs and its role in diagnosis and prognosis of breast cancer [6]. A CTC-based liquid biopsy enables non-invasive monitoring of drug resistance. Balakrishnan et al. [7] describe current methods for isolation and expansion of CTCs. The low number of CTCs in peripheral blood demands an ex-vivo expansion. The review details the importance of tumor cell plasticity in CTC isolation and emphasizes the need to establish a method which does not require prior enrichment of CTCs. The deeper characterization of these rare cell populations is essential for clinical utility and management of cancers [7]. Effective isolation and expansion of CTCs is the key to develop a functional assay which could be used for patient-specific treatment strategies. Assessment of gene expression profiles in CTCs sheds additional light on their heterogeneity and potential functionality in tumorigenesis and metastasis. Hassan et al. [8] review recent studies assessing gene expression profiles of CTCs. Several studies have employed different molecular platforms such as RT-qPCR, RNA-ISH and RNAseq to identify a CTCbased gene signature [8]. Recent advances in sequencing techniques have allowed researchers and clinicians to profile DNA, RNA and proteins at molecular levels to discover novel cancer drivers and therapeutic targets. Oncogenes and tumor suppressor genes are the major regulators of gene transcription, transition due to insertion or a deletion (indel), rearrangements or single nucleotide mutations. Walavalkar et al. [9] highlight the different kind of variations within the enhancers and the effect of these variations in cancer initiation. Sadasivam et al. [10] provide an indepth description of the identification of cancer stem cells (CSCs) in oral cancers and the molecular features specific to oral CSCs. Sala et al. [11] provide a comprehensive review on the role of argonaute proteins (AGO) in cellular processes. The review primarily focuses on the canonical as well as non-canonical functions of AGO proteins in mammals. In addition, they also focused on the association of AGO proteins in cell invasion, survival and poor prognosis in cancer [11].

The complex interaction between the tumor microenvironment and cancer cells is one of the major determinants in tumor progression and metastasis. Proteoglycans are the major macromolecules present in the ECM, playing a crucial role in angiogenesis, proliferation, invasion, and metastasis. Singh et al. [12] review the role of arylsulfatase, an enzyme which mediates desulfation of proteoglycans in oncogenesis. This review also depicts the role of proteoglycan sulfatases in EMT and remodeling of ECM, further suggesting sulfatases as targets for therapeutic interventions [12]. Cancer-associated fibroblasts (CAFs) are an abundant stromal cell type found in the tumor microenvironment (TME) and their reciprocal interaction with tumor cells will lead to the remodeling of the ECM, thus promoting intravasation of tumor cells. Patel et al. [13] summarize the heterogenous origin of CAFs and their regulation in tumor progression. Normal fibroblasts are converted to CAFs through the growth factors TGF $\beta 1$, SDF1 and PDGFR $\beta$ secreted from tumor cells. CAFs also induce EMT through paracrine signaling and facilitate tumor growth by secreting growth factors and cytokines/chemokines [13].

Metastasis, which causes most cancer deaths, is a major challenge in translational advances. Understanding the molecular mechanisms underlying metastatic progression of disease will improve metastasis-oriented drug development strategies. In this issue, we integrated the current knowledge on various aspects of metastasis and the significance of multimodal liquid biopsy tools. This introduction has provided an overview of the utility of the different approaches described in these collected papers to deepen our understanding of the mechanisms of drug resistance, tumor progression and metastatic processes.

\section{Author contributions}

PK conceptualized the draft. IAG and JS were involved in original draft preparation. PK was involved in the final revision and edit of the editorial.

\section{Ethics approval and consent to participate}

Not applicable.

\section{Acknowledgment}

Not applicable.

\section{Funding}

This research received no external funding.

\section{Conflict of interest}

The authors declare no conflict of interest. PK is serving as one of the Guest editors of this journal. We declare that PK had no involvement in the peer review of this article and has no access to information regarding its peer review. Full responsibility for the editorial process for this article was delegated to GP. 


\section{References}

[1] Moller A. The role of exosomes in the promotion of epithelial-to-mesenchymal transition and metastasis. Frontiers in Bioscience-Landmark. 2020; 25: 1022-1057.

[2] Carvalho AS, Baeta H, Silva BC, Moraes MCS, Bodo C, Beck $\mathrm{HC}$, et al. Extra-cellular vesicles carry proteome of cancer hallmarks. Frontiers in Bioscience-Landmark. 2019; 25: 398-436.

[3] Dhar R. MTiness in pseudo-malignant behavior of trophoblasts during embryo implantation. Frontiers in Bioscience-Landmark. 2021; 26: 717-743.

[4] Roy S. EMT imparts cancer stemness and plasticity: new perspectives and therapeutic potential. Frontiers in BioscienceLandmark. 2021; 26: 238-265.

[5] Raja R, Pandey A, Kumar P. Epithelial to mesenchymal plasticity: role in cancer progression. Frontiers in BioscienceLandmark. 2019; 25: 838-873.

[6] Kumar LD. EMT in breast cancer metastasis an interplay of microRNAs signaling pathways and circulating tumor cells. Frontiers in Bioscience-Landmark. 2020; 25: 979-1010.

[7] Kumar P. Circulating tumor cells as an emerging tool in cancer therapy. Frontiers in Bioscience-Landmark. 2020; 25: 606-631.

[8] Hassan S. Applications of RNA from circulating tumor cells. Frontiers in Bioscience-Landmark. 2020; 25: 874-892.

[9] Notani D. Beyond the coding genome non-coding mutations and cancer. Frontiers in Bioscience-Landmark. 2020; 25: 18281838.
[10] Sadasivam S. A perspective on challenges and opportunities in characterizing oral cancer stem cells. Frontiers in BioscienceLandmark. 2020; 25: 1011-1021.

[11] Vidigal JA. AGO unchained Canonical and non-canonical roles of Argonaute proteins in mammals. Frontiers in BioscienceLandmark. 2020; 25: 1-42.

[12] Singh V, Bhat R. Proteoglycan desulfation: a critical step in oncogenesis? Frontiers in Bioscience-Landmark. 2019; 25: 760-780.

[13] Singh PS. Cancer associated fibroblasts phenotypic and functional heterogeneity. Frontiers in Bioscience-Landmark. 2020 25: 961-978.

Send correspondence to: Prashant Kumar, Institute of Bioinformatics, International Technology Park, 560066 Bangalore, Karnataka, India, Manipal Academy of Higher Education (MAHE), 576104 Manipal, Karnataka, India, Somaiya Institute of Research and Consultancy (SIRAC), Somaiya Vidyavihar University (SVU), 400077 Mumbai, Maharashtra, India, E-mail: prashantkumar@somaiya.edu ${ }^{\dagger}$ These authors contributed equally. 